\title{
Effect of microwave frequency on dielectric properties of oil palm shell (OPS) and OPS char synthesized by microwave pyrolysis of OPS
}

\author{
Manoj Tripathi a , J.N. Sahu ${ }^{\text {b,c,*, P. Ganesan }}{ }^{\mathrm{a}, * *}$, Purushothaman Monash ${ }^{\mathrm{c}}$, T.K. Dey ${ }^{\mathrm{d}}$ \\ ${ }^{a}$ Department of Mechanical Engineering, Faculty of Engineering, University of Malaya, 50603 Kuala Lumpur, Malaysia \\ b Petroleum and Chemical Engineering Programme Area, Faculty of Engineering Institut Teknologi Brunei, Tungku Gadong. P. O. Box 2909, Brunei \\ Darussalam \\ 'Department of Chemical Engineering, Faculty of Engineering, University of Malaya,50603 Kuala Lumpur, Malaysia \\ ${ }^{\mathrm{d}}$ Thermophysical Measurement Laboratory, Cryogenic Engineering Centre, Indian Institute of Technology, Kharagpur 721302, WB, India
}

A R T I C L E I N F O

\section{Article history:}

Received 1 December 2014

Received in revised form 9 January 2015

Accepted 12 January 2015

Available online 14 January 2015

Keywords:

Dielectric properties

Microwave heating

Oil palm shell (OPS)

OPS char

Penetration depth
A B S T R A C T

Microwave heating is considered to be a potential, efficient and fast method for thermal treatment of biomass. Therefore, elemental understanding of dielectric properties of biomass is necessary before irradiating it with microwave radiations. Dielectric properties along with penetration depth, relaxation time and static permittivity of oil palm shell (OPS) and OPS char were investigated in the frequency ranging between 0.5 and $20 \mathrm{GHz}$ at room temperature. At low frequencies, dielectric constant of OPS was higher than that of OPS char while at higher frequencies, vice versa is observed. Penetration depth for OPS char was higher than that of OPS at low frequencies. Significant variation in relaxation time was observed for OPS in different frequency range, suggesting that the OPS do not obey the Debye equation for dielectrics. Relaxation time and static permittivity for OPS was higher as compared to OPS char.

(c) 2015 Elsevier B.V. All rights reserved.

\section{Introduction}

Malaysia produces more than $52 \%$ of the total palm oil across the world. Total area coverage of palm trees in Malaysia is about 3.8 million hectare and is expected to reach to 5 million hectare by year $2020[1,2]$. This huge amount of palm oil production also involves a large production of oil palm shell (OPS) waste. In 2007 the oil palm waste was 4.7 million ton which increased to 5.2 million ton by year 2009 [3]. Efficient and economical utilization of OPS will help significantly to reduce the waste. OPS have been a research interest, since, it can be very useful in construction industry. Moreover, overuse of virgin metals such as granite aggregate and mining sand at present by the industry has created an ecolog ical imbalance. Agricultural wastes including OPS are considered to be a potential replacement of conventional granite aggregate [4]. Production of bio oil and other value added products like bio char using different thermochemical techniques is one of the com-

* Corresponding author at: University of Malaya, Department of Chemical Engineering, Faculty of Engineering, 50603 Kuala Lumpur, Malaysia. Tel.: +60 37967 5204/7670; fax: +60 379674579 .

** Corresponding author.

E-mail addresses: jay_sahu@yahoo.co.in (J.N. Sahu), poo_ganesan@um.edu.my (P. Ganesan).

http://dx.doi.org/10.1016/j.jaap.2015.01.007

0165-2370/O 2015 Elsevier B.V. All rights reserved. monly practiced ways of utilizing OPS [5-7]. Microwave pyrolysis of biomass has emerged as a very promising technique to convert biomass to bio oil and other products. Bio oil produced from OPS has low power generation ability as compared to fossil fuels, however, with a further processing, it can be utilized as fuel [8]. Bio char produced in the process has various applications in different areas, e.g., can be used for soil procurement. Bio char enhances the natural rate of carbon sequestration in soil and inhibits the decomposition rate of nutrients which finally results in the increase of soil quality $[9,10]$. Bio char is considered to have remarkable environmental advantages over fossil fuels [11]. Bio char obtained can be utilized as bio modifier for asphalt cement as discussed in Zhao et al. [12] In addition, it can be used in purification industry to remove heavy metals such as $\mathrm{Pb}$ [13], $\mathrm{Hg}$ [14], Cd [15], $\mathrm{Cr}$ [16], Zn [17] and $\mathrm{Ni}$ [18]. and purification of chemicals like tetracycline [19] and phenol [20].

Microwave heating is considered to be a potential alternative for conventional heating techniques which is either based on conduction or convection. Both of these processes has disadvantages of heat loss (in other words energy loss) to the surroundings; while in microwave heating, the material can be heated directly that minimizes the heat loss to the surroundings. As a result, microwave heating is more economical [21,22] and provides a fast and uniform heating as compared to conventional heating [23,24]. Microwave radiation is in between radiofrequency waves and infrared waves in the spectrum of electromagnetic radiations with the frequency 
ranging from $300 \mathrm{MHz}$ to $300 \mathrm{GHz}$ which corresponds to wavelength ranging between $1 \mathrm{~m}$ and $1 \mathrm{~mm}$. Microwave heating has been found to be useful in accelerating chemical reactions and wide microwave heating applications $[25,26]$. Microwave heating is frequently being used in food processing, material treatment (curing and preheating of ceramics, molding and treatment of plastics and rubbers), and wood drying [27].

The use of microwave heating for different types of biomass such as rice straw, sugarcane bagasse, wheat straw, empty fruit bunch and other agricultural wastes [28-33]. Navarrete et al. [34] studied the dielectric properties of Rosemary at $915 \mathrm{MHz}$ and $2450 \mathrm{MHz}$ microwave frequency at elevated temperatures and found that the dielectric constant decreases at a high frequency. A similar finding was reported by Motasemi et al. [35] on the dielectric measurement of switchgrass. Zhu et al. [36-39] used domestic microwave to preheat rice straw and wheat straw and this method is found to be very effective in fermentation and enzymatic hydrolysis. The intrinsic properties of biomass highly affect the biomass conversion process and also determine the process complexities [40]. Different materials behave differently when exposed to microwave radiation depending on dielectric properties of the material [41]. Hence, before using microwave for heating applications, one must have elementary understanding of the material interaction with microwave. Microwave heating depends upon the dielectric properties of the material. Therefore, to make the microwave heating more efficient it is important to know the dielectric properties, especially dielectric constant $\left(\varepsilon^{\prime}\right)$, dielectric loss $\left(\varepsilon^{\prime \prime}\right)$ and tangent loss factor $(\tan \delta)$, of the material. In the present study, dielectric properties of OPS and OPS char are investigated at frequencies ranging between 0.5 and $20 \mathrm{GHz}$. A wide range of frequency selected in this present work is to get a clear understanding of material interaction and to evaluate the optimum frequency for better microwave heating. Also this will be very useful to find the amount of energy absorbed by the biomass. Moreover, relaxation time and penetration depth of the materials under investigation have also been studied for the above mentioned frequency range because these parameters also plays an important role in the optimization of microwave heating process of any material. Furthermore, the dielectric properties of OPS and OPS char have also been measured at $915 \mathrm{MHz}$ and $2450 \mathrm{MHz}$ frequencies $(32.76 \mathrm{~cm}$ and $12.23 \mathrm{~cm}$, respectively). These frequencies are selected because most of the domestic and industrial instruments are tuned to be operated at these frequencies [42].

\section{Materials and methods}

The OPS used in the present study was supplied by Seri Ulu Langat Palm Oil Mill Dengkil, Selangor, Malaysia. The purchased OPS particles were brown in color and in granular forms. OPS char was prepared by microwave pyrolysis of the OPS and used in the present investigation.

\subsection{Preparation of OPS char}

The OPS was washed by normal water to remove dust and water soluble impurities. Then it was dried in an oven at $80^{\circ} \mathrm{C}$ for $24 \mathrm{~h}$. The dried OPS was then crushed manually and sieved to a particle size of less than $150 \mu \mathrm{m}$. This sieved OPS was pyrolyzed by HAMiabC1500 microwave muffle system oven containing a microwave generator with a microwave power controller. $100 \mathrm{~g}$ of OPS was filled in to a cylindrical quartz tube ( $\mathrm{I} . \mathrm{D}=28 \mathrm{~mm}, 0 . \mathrm{D}=35 \mathrm{~mm}$ and $L=500 \mathrm{~mm}$ ). The OPS filled quartz tube was exposed to microwave radiation at $1000 \mathrm{~W}$ for 30 min under inert atmosphere maintained by a constant nitrogen flow rate of $400 \mathrm{~cm}^{3} / \mathrm{min}$. The quartz tube was allowed to cool down to room temperature. After cooling, the

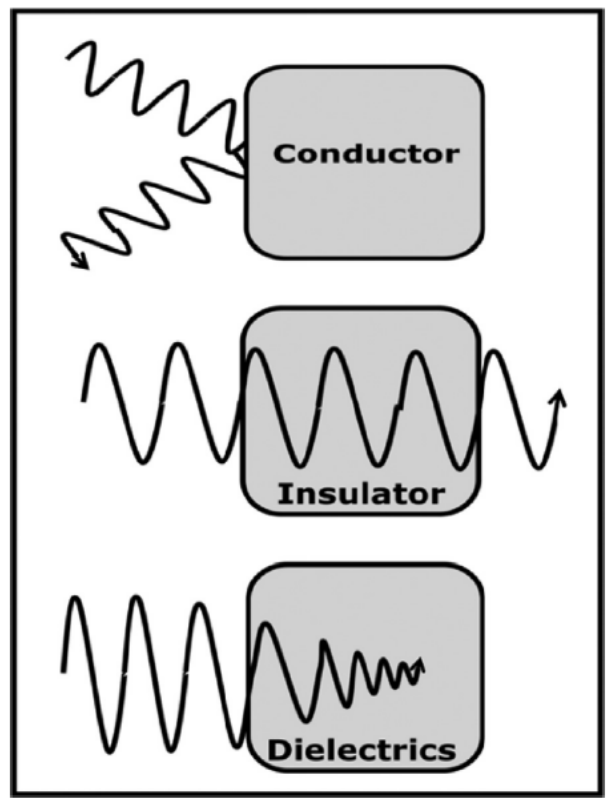

Fig. 1. Interaction of different materials with microwave radiation.

material was collected from the quartz tube and stored in air tight containers. This microwave treated OPS is termed as OPS char.

\subsection{Sample preparation and dielectric measurement}

OPS and OPS char were grinded into a powder form. Then this powdered material was formed into cylindrical pellets using a cylindrical die with an inner diameter of $20 \mathrm{~mm}$. A pressure of $450 \mathrm{kN} / \mathrm{m}^{2}$ was applied on the stainless steel die using a hydraulic press machine to convert the powdered material into cylindrical pellets. These pellets were used for the dielectric measurements.

\subsection{Dielectric measurement}

Dielectric measurement of the OPS and OPS char was carried out using HP 85070B open-ended coaxial probe which was attached to computer controlled HP 8722D vector network analyzer (VNA). The sample was brought to contact with the analyzing probe to measure the dielectric properties. The dielectric measurements were carried out at frequencies between 0.5 and $20 \mathrm{GHz}$ at room temperature to see the effect of frequency on the dielectric properties.

\section{Mathematical formulations}

Efficiency of microwave heating depends upon the absorption of microwave radiation by the material. Different materials behave differently when exposed to microwave radiation. Fig. 1 represents the classification of materials on the basis of their behavior on interaction with the microwave radiation. The materials which do not interact with incident microwave radiation and reflect it back are termed as conductors. Those materials which let the microwave radiation pass through without absorbing it, are called insulators. Dielectrics, which is the third category of materials, allow the incident microwave radiation to enter within and absorb it [43]. For effective microwave heating, the material should be able to absorb 
the microwave radiation and the ability of absorbing the microwave radiation is determined by the dielectric properties of the material.

Absolute dielectric constant $(\epsilon)$ is composed of a real part and imaginary parts and is given by Eq. (1) [44]:

$\epsilon=\epsilon^{\prime}-j \epsilon^{\prime \prime}$

where, $\epsilon^{\prime}$ is the real part of the complex function and formally called dielectric constant, $j^{2}=-1$ is the imaginary unit and $\epsilon^{\prime \prime}$ is the imaginary part which is referred to dielectric loss. $\epsilon^{\prime}$ value of the material represents its ability to store the electric energy within it. The imaginary part represents the dissipation (or loss) of energy within the material.

The complex dielectric constant is often represented in Argand plane. Argand plane is a curve with $\epsilon^{\prime}$ on abscissa and $\epsilon^{\prime \prime}$ on ordinate having frequency as parameter. If we join any point of this curve to the origin it gives us a complex conjugate of complex dielectric constant $\epsilon$ as shown in Eq. (2) [44]:

$\epsilon^{*}=\epsilon^{\prime}+j \epsilon^{\prime \prime}$

where $\epsilon^{*}$ is the complex conjugate of $\epsilon$. If joining of a point to the origin makes an angle $\delta$ with the abscissa and is given by Eq. (3) [45]:

$\tan \delta=\frac{\epsilon^{\prime}}{\epsilon^{\prime \prime}}$

where, the angle $\delta$ is known as loss angle and $\tan \delta$ is known as tangent loss. Tangent loss determines the ability of the material to convert the electromagnetic waves into heat for a specific frequency at a fixed temperature [44].

\subsection{Penetration depth}

Penetration depth $\left(D_{\mathrm{P}}\right)$ is a very important criterion for designing and scaling up of any microwave heating system. It not only depends upon characteristics of the heating material but also depends on the frequency of microwave radiation. Penetration depth represents how deep microwave radiation penetrates a material and it is calculated using Eq. (4) [46].

$D_{P}=\frac{\lambda_{0}}{2 \pi\left(2 \epsilon^{\prime}\right)^{0.5}}\left[1+\left(\frac{\epsilon^{\prime \prime}}{\epsilon^{\prime}}\right)^{2^{0.5}}-1\right]^{-0.5}$

where $\lambda_{0}=c / f$ refers to the wavelength of microwave in free space, $c$ is the velocity of light in free space and $f$ is the frequency of microwave radiation

\subsection{Relaxation time}

When an electromagnetic field is applied to a material, it changes the orientation of the dipoles along the direction of electric field and upon removal of the field dipoles come back to their original orientation. Relaxation time $(\tau)$ is the time taken by the dipoles to come to their original orientation on removing the field. Eq. (5) is used to calculate the relaxation time [44].

$\epsilon^{\prime}=\left(-\omega \epsilon^{\prime \prime}\right) \tau+\epsilon_{s}$

where $\omega$ is angular frequency of microwave and $\epsilon_{\mathrm{s}}$ is static permittivity. If we plot a curve between $\epsilon^{\prime}$ versus $-\omega \epsilon^{\prime \prime}$ then the slope of the curve will be equal to $\tau$ and the intercept of the line on $\epsilon^{\prime}$ axis will equal to the $\epsilon_{\mathrm{s}}$.

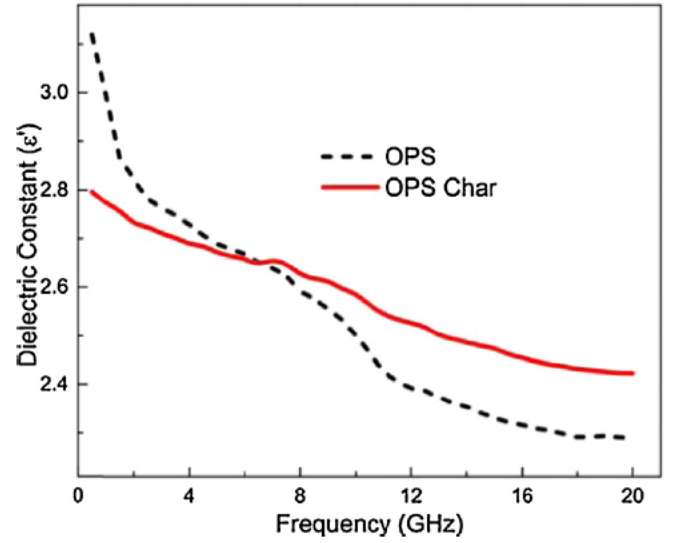

Fig. 2. Dielectric constant of OPS and OPS char as function of frequency at room temperature.

\section{Results and discussion}

\subsection{Effect of microwave frequency on dielectric properties}

Fig. 2 represents the variation in the dielectric constant $\left(\epsilon^{\prime}\right)$ of OPS and OPS char as a function of frequency at room temperature and it shows that dielectric constant for OPS and OPS char decrease with an increase of the frequency. Value of $\epsilon^{\prime}$ is reduced by $26.6 \%$ and $13.3 \%$ for OPS and OPS char, respectively. For OPS, the dielectric constant dropped sharply for microwave frequency ranging from $0.5 \mathrm{GHz}$ up to $2 \mathrm{GHz}$. For OPS char, the $\epsilon^{\prime}$ value decreased with nearly linear trend throughout the observed frequency range. The decrease in the dielectric constant at higher frequencies is caused by the drop in polarization, since, at higher frequency, orientational polarization mechanism ceases to contribute to net polarization [47]. Also when the frequency of microwave radiation increases, the direction of the field changes very frequently and the dipoles does not get enough time to orient themselves in the direction of the field which reduces the polarization of the material and consequently the dielectric constant reduces.

Fig. 2 also reveals that for low frequencies $(<5 \mathrm{GHz})$ the dielectric constant of OPS is higher than that of OPS char. This can be explained on the basis of moisture content in the materials. OPS char has lower moisture content as compared to OPS since moisture is removed during the formation of the OPS char. Materials with more moisture content show high dielectric constant value as reported in many studies $[45,48,49]$. Water is a good absorber of microwave; High moisture ensures a better absorption of microwave radiation and result in a better dielectric constant value. At high frequencies the $\epsilon^{\prime}$ of OPS is lesser than that of OPS char.

Tangent loss for OPS and OPS char has been calculated using Eq. (3). The variation of the dielectric loss $\left(\epsilon^{\prime \prime}\right)$ for OPS and OPS char with the frequency at room temperature is shown in Fig. 3(a). In general, $\epsilon^{\prime \prime}$ for both the materials increases with the increase of the frequency but the increase is significant for OPS char and less significant for OPS. At lower frequencies, i.e., from $0.5 \mathrm{GHz}$ to $3.5 \mathrm{GHz}$ $\epsilon^{\prime \prime}$ value is higher for OPS than that for OPS char. However, this is vice versa above $3.5 \mathrm{GHz}$; $\epsilon^{\prime \prime}$ values of OPS are distributed nonuniformly but in a flat trend. A bell shape curve is a commonly found in $\epsilon^{\prime \prime}$ versus frequency plot for most materials [44]. A bell shape curve is slightly seen between $4.5 \mathrm{GHz}$ and $13 \mathrm{GHz}$ for OPS and $7 \mathrm{GHz}$ and $14 \mathrm{GHz}$ for OPS char. Fig. 3(a) also shows that at

\section{Link to Full-Text Articles :}

http://ac.els-cdn.com/S0165237015000194/1-s2.0-S0165237015000194-main.pdf? tid=048de4b2-f3a2-11e4b8d7-00000aacb360\&acdnat $=143088397135 e 345 e 517 e 87 d 9 b 2267 a 72 d c 1 d 34324$ 